Case

\title{
Does Gefitinib Have Effects on EGFR Mutation-Positive Thymoma? - Case Report of Thymoma Recurrence
}

\author{
Tomoyuki Nakagiri, MD, PhD,${ }^{1}$ Soichiro Funaki, MD, PhD,${ }^{1}$ Yoshihisa Kadota, MD, PhD,${ }^{2}$ \\ Yukiyasu Takeuchi, MD, $\mathrm{PhD},{ }^{3}$ Hiroyuki Shiono, MD, $\mathrm{PhD},{ }^{4}$ Akinori Akashi, MD, $\mathrm{PhD},{ }^{5}$ \\ and Meinoshin Okumura, $\mathrm{MD}, \mathrm{PhD}^{1}$
}

\begin{abstract}
A 56-year-old woman heavily pretreated for a thymoma was referred to our hospital with recurrence. Following additional surgery, the tumor recurred again in the left thoracic cavity. Because of previous multiple operations and repeated chemotherapies, the patient was considered unable to tolerate additional surgery or chemotherapy. After we obtained positive evidence of epidermal growth factor receptor (EGFR) mutation, she was administrated gefitinib for 3 months. Although immunohistochemistry findings showed that the tumor was EGFR positive, gefitinib therapy led no reduction of the tumor size. After undergoing immunotherapy, the patient suffered from repeated occurrences of pneumonia, and died from respiratory failure.
\end{abstract}

Keywords: thymoma, epidermal growth factor receptor (EGFR) mutation, EGFR tyrosine kinase inhibitor

\section{Introduction}

Kurup, et al. presented results of a phase II study of gefitinib for advanced thymic malignancies at an American

${ }^{1}$ Department of General Thoracic Surgery, Osaka University Graduate School of Medicine, Suita, Osaka, Japan

${ }^{2}$ Department of Thoracic Surgery, Osaka Prefectural Medical Center for Respiratory and Allergic Diseases, Habikino,Osaka, Japan

${ }^{3}$ Department of General Thoracic Surgery, National Hospital Organization Toneyama Hospital, Toyonaka, Osaka, Japan

${ }^{4}$ Department of General Thoracic Surgery, Nara Hospital, Kinki University School of Medicine, Ikoma, Nara, Japan

${ }^{5}$ Department of General Thoracic Surgery, Takarazuka Municipal Hospital, Takarazuka, Hyogo, Japan

Received: June 12, 2013; Accepted: October 15, 2013

Corresponding author: Tomoyuki Nakagiri, MD, PhD. Department of General Thoracic Surgery, Osaka University Graduate School of Medicine, 2-2 (L5) Yamadaoka, Suita, Osaka 565-0871, Japan

Email: gilly64@thoracic.med.osaka-u.ac.jp

(C)2014 The Editorial Committee of Annals of Thoracic and Cardiovascular Surgery. All rights reserved.
Society of Clinical Oncology (ASCO) meeting in 2005. They reported that epidermal growth factor receptor (EGFR) mutations do not appear to be common in thymic malignancies and gefitinib was well tolerated in those cases, with minimal activity in a heavily pretreated population. However, the single partial remission (PR) patient in their study had no evidence of EGRF mutation, while the cohort included thymoma and thymic carcinoma cases. Thus, the effects of gefitinib against thymoma with EGFR mutation remain unknown. Here, we report a heavily pretreated patient with an EGFR mutation positive thymoma who underwent gefitinib therapy.

\section{Case Report}

A 56-year-old woman was referred to our institution in 2006 for therapy for a recurrent thymoma. In 1995, she was diagnosed with a thymoma and underwent a thymectomy under video assisted thoracic surgery after steroid pulse therapy. The mass was $3 \times 3 \times 2 \mathrm{~cm}$ in size and 


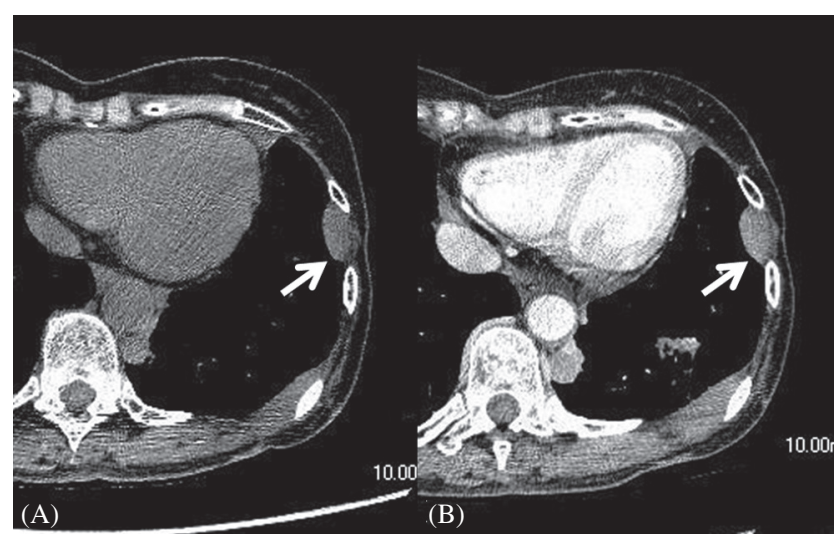

Fig. 1 (A) Chest computed tomography (CT) image showing thymoma recurrence in left thoracic cavity (arrow). (B) CT image obtained after 3 months of gefitinib therapy showing no effects, resulting in no reduction of the disease (arrow).

showed invasion to the right pleura (type B2, Masaoka stage II, T2 N0 M0, stage II). After that resection, she underwent radiation therapy to the mediastinum (50 Gy) and was followed as an outpatient. In 2000, recurrences in the bilateral thoracic cavities and mediastinum were found, and she underwent systemic chemotherapy with cisplatin (CDDP), adriamycin (ADR), and cyclophosphamide (CPA), with the effect of no reduction of the disease. Therefore, a left upper lobectomy with rib resection and right tumor resection as much as possible were performed, along with diaphragm resection and an intraarterial chemotherapy regimen (CDDP, ADR, CPA, vincristine; PR), because the tumor remained in the mediastinum. Thereafter, she underwent chemotherapy (carboplatin, paclitaxel) for another recurrence, though the effect was no reduction of the recurrence. She was then introduced to our hospital for additional therapies.

In 2006, we performed mediastinal tumor enucleation for recurrence at our hospital. The next year, she underwent tumor enucleations twice for tumor dissemination in the thoracic cavity. In 2009, other recurrences in the left thoracic cavity were noted (Fig. 1A). However, because she had already undergone repeated operations and chemotherapies, the patient did not seem able to tolerate another surgery or more chemotherapy based on respiratory function and performance status.

A tumor sample from the most recent operation was subjected to fragment analysis (SRL, Inc., Tokyo, Japan), which revealed an EGFR mutation (exon19; E746-A750 deletion). We then administrated gefitinib (250 mg/day)

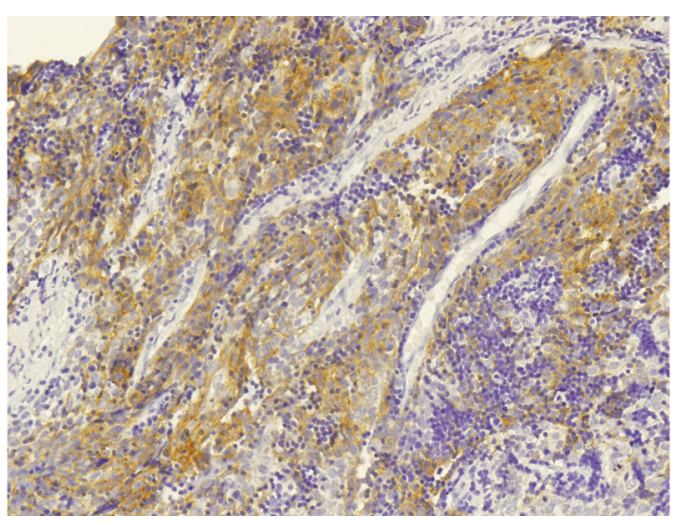

Fig. 2 Immunohistochemistry findings showing epidermal growth factor receptor (EGFR) as brown stained structures in epithelial thymoma cells. for 3 months using the public funds of our hospital. She spent the 3 months without complication. Although pathological reassessment of the tumor sample detected expression of EGFR in immunohistochemistry findings (Fig. 2), computed tomography performed 3 months after administration showed that the effect was no reduction of the tumor (Fig. 1B) and gefitinib therapy was stopped. Immunotherapy was then performed, though recurrence causing stenosis in the right upper bronchus was observed and obliteration of the right upper bronchus showed gradual deterioration. After suffering from repeated pneumonia occurrences, the patient died from respiratory failure at the age of 62 in the emergency room of another hospital.

\section{Discussion}

Previous reports and reviews have described the relationship between EGFR and thymoma. ${ }^{1-5)}$ However, an EGFR mutation in a thymoma is rare, with only about $10 \%$ of Japanese patients reported to possess that. ${ }^{2,3)} \mathrm{In}$ addition, there are reports of administration of an EGFR tyrosine kinase inhibitor (TKI) for thymoma. ${ }^{4)}$ However, there are no reports regarding the effects of EGFR-TKI against an EGFR mutation positive thymoma.

In their ASCO presentation, Kurup, et al. concluded that gefitinib for malignant thymic tumors was well tolerated. ${ }^{1)}$ In contrast, in a review, Kelly, RJ et al. stated that, at present, therapies with EGFR TKI for patients with thymic malignancies could not be recommended.5) However, when considering EGFR TKI for lung cancer and its 
exclusive effect on only EGFR mutation positive tumors, both of those opinions lack convincing evidence. It can also be speculated that because EGFR mutations are extremely rare in thymic tumors, the effect of EGFR TKI is poor. On the other hand, Yoh, et al. proposed that patients with thymomas harboring EGFR mutations may profit from therapy with EGFR TKI. ${ }^{2}$

Although the thymoma in our patient possessed the EGFR mutation, EGFR TKI did not reduce the tumor sizes. Our patient can be classified as "heavily pretreated" and the EGFR mutation was not detected until the latest sample was obtained prior to beginning EGFR TKI therapy. In addition, EGFR expression was detected in that specimen. Yoh, et al. also speculated that an EGFR mutation may not be implicated in the carcinogenesis of a thymoma based on the frequency of EGFR mutations in such cases. ${ }^{2)}$ Our results support that speculation based on the lack of tumor reduction with EGFR TKI for a thymoma with the EGFR mutation in the present patient.

On the other hand, it can also be said that the EGFR TKI was effective for our patient. We stopped the therapy for only 3 months along the informed consent to the patient, on which when the therapy was effective for the tumor in 3 months, the therapy was continued after the 3 months, because EGFR TKI was off-label use for thymoma in Japan, and the therapy did not reduce the tumor size. Although, tumor reduction was not seen in the patient, the tumor size was stable during the therapy without complication.
This is the first report regarding the effects of EGFR TKI therapy for a patient, who was heavily pretreated, with an EGFR mutation positive thymoma. Accumulation of reports of EGFR TKI therapy for mutation positive cases, as well as investigations with a focus on such cases and basic studies are expected to increase knowledge of this topic.

\section{Disclosure Statement}

The authors have no conflicts of interest to disclose.

\section{References}

1) Kurup A, Burun M, Dropcho S, et al. Phase II study of gefitinib treatment in advanced thymic malignancies. J Clin Oncol 2005; 23: 7068.

2) Yoh K, Nishiwaki Y, Ishii G, et al. Mutational status of EGFR and KIT in thymoma and thymic carcinoma. Lung Cancer 2008; 62: 316-20.

3) Suzuki E, Sasaki H, Kawano O, et al. Expression and mutation statuses of epidermal growth factor receptor in thymic epithelial tumors. Jpn J Clin Oncol 2006; 36: 351-6.

4) Palmieri G, Marino M, Salvatore M, et al. Cetuximab is an active treatment of metastatic and chemorefractory thymoma. Front Biosci 2007; 12: 757-61.

5) Kelly RJ, Petrini I, Rajan A, et al. Thymic malignancies: from clinical management to targeted therapies. J Clin Oncol 2011; 29: 4820-7. 